\title{
Antenatal diagnosis of Double chambered Left Ventricle. Post-natal evolution to LV dysfunction
}

\author{
Guy Vaksmann ${ }^{1}$, Elodie Coudoux ${ }^{2}$, and Angelina Dubois ${ }^{2}$ \\ ${ }^{1}$ Hopital Prive La Louviere \\ ${ }^{2}$ Centre Hospitalier de Calais
}

April 13, 2021

\begin{abstract}
Double chambered left ventricle is an exceedingly rare congenital anomaly. We report a case diagnosed prenatally at 24 weeks of gestation and its postnatal evolution to left ventricular dysfunction.
\end{abstract}

Antenatal diagnosis of Double chambered Left Ventricle. Post-natal evolution to LV dysfunction.

Guy Vaksmann ${ }^{1}$ M.D., Elodie Coudoux ${ }^{2}$, M.D., Angelina Dubois ${ }^{3}$, M.D.

1. Department of Pediatric cardiology, Hôpital privé de La Louvière, Lille, France

2. Department of Obstetrics, Centre hospitalier de Calais, Calais, France

3. Department of Neonatology, Centre hospitalier de Calais, Calais, France

Corresponding author: Dr Guy Vaksmann, Hôpital Privé de la Louvière, 69 rue de La Louvière 59000, Lille France. FAX: Email: Guy.vaksmann@wanadoo.fr

Shortened Title: Double Chambered Left Ventricle

Antenatal diagnosis of Double chambered Left Ventricle - Post-natal evolution to LV dysfunction.

Abstract : Double chambered left ventricle is an exceedingly rare congenital anomaly. We report a case diagnosed prenatally at 24 weeks of gestation and its postnatal evolution to left ventricular dysfunction.

Key words: Double Chambered Left Ventricle; Antenatal diagnosis; Fetal Echocardiography.

Introduction: Double Chambered Left Ventricle (DCLV) is an exceedingly rare congenital malformation which there are 2 types according to the relationship between the main chamber (MC) and the accessory chamber $(\mathrm{AC})$ of the left ventricle $(\mathrm{LV})$. In type $\mathrm{A}, \mathrm{MC}$ and $\mathrm{AC}$ are in a superior-inferior arrangement. In type $\mathrm{B}$, the rarer, $\mathrm{MC}$ and $\mathrm{AC}$ are parallel to each other and lie side by side [1]. We report a new case of type B DCLV diagnosed antenatally in whom the post-natal evolution was characterized by occurrence of LV dysfunction.

Case report: A 38 year-old primigravida woman was referred at 24 weeks of gestation because of abnormal fetal heart on routine obstetric ultrasound screening. No other anomaly was suspected on echography.

Fetal echocardiography showed that the left ventricle (LV) was divided into 2 cavities of equal size that lie side by side (figure 1, video 1). The main chamber (MC) was in connection with mitral and aortic valves (figure 2, video 2). The aneurysm-like accessory chambers (AC) was large and connected to the LV through a wide communication in the lateral wall. The 2 chambers were separated by muscle bundles. 
M-mode echocardiogram demonstrated that the AC contracted synchronously with the MC and the RV characterizing a DCLV instead of a left ventricular aneurysm (figure 3). Color doppler showed that filling of $\mathrm{AC}$ occurred in diastole and draining toward MC during systole (Figure 4).

Amniocentesis with CGH-array analysis was normal. The mother gave birth to a female infant weighting 2.8 $\mathrm{kg}$ at 37 weeks of gestation. The cardiovascular findings suspected prenatally were confirmed after birth by cross-sectional echocardiography performed on day 1 (figure 5, video 3). The newborn was asymptomatic. Her EKG showed inverted T waves in leads V3 to V6. There was no intraventricular conduction delay.

During subsequent visits, LV dilated progressively, and LV function decreased (figure 6, videos 4). There was a mild mitral insufficiency. It prompted introduction of a treatment with captopril and carvedilol at 2 months of age. At age 8 months the child is still asymptomatic on beta blockers and ACE inhibitor. Physical examination is normal.

\section{Discussion:}

Our case illustrates a type B DCLV and is the first case in which antenatal images are available.

Differential diagnosis includes diverticulum or aneurysm of the left ventricle. In the former the outpouching has 3 layers, contracts during systole and has a narrow connection with the LV. In the later, connection with the LV is large and the cavity does not contract or is dyskinetic [2]. In our patient the outpouching had a wide connection to the LV from which it was separated from muscle bundles but had initially a normal contractility characterizing DCLV.

Several authors consider that type B DCLV is a benign condition frequently found incidentally [1;3-6]. It can also be complicated by dysrhythmias [7] or by left ventricular dysfunction as in our case [8]. It is likely that the size of the AC influences the long-term tolerance of the anomaly since a large AC causes chronic volume overload of the left ventricle that could lead to LV dysfunction. Indeed, in a report of more than 800 cases of left ventricular aneurysm or diverticulum Ohlow et al. noticed that LV dysfunction occurred in the largest outpouchings [9].

Thus, type B DCLV is an exceedingly rare congenital heart malformation that can be diagnosed during fetal life. Although most of reported cases have a benign outcome and are asymptomatic, its prognosis probably depends on the size of the AC, large AC could result in chronic LV overload and dysfunction. Prenatal family counselling should mention this possible evolution.

\section{References}

1- Zhang X, Wang J, Cao Y, et al. Diagnosis of double-chambered left ventricle using echocardiography. Echocardiography $2020 ; 37: 1095-1100$.

2- Marijon E, Ou P, Fermont L, et al. Diagnosis and outcome in congenital ventricular diverticulum and aneurysm. J Thorac cardiovasc surg . $2006 ; 131$ :433-437.

3- Bilici M, Demir F, Akin A. Echocardiographic diagnosis of double-chambered left ventricle. J Echocardiogr $2016 ; 14: 176-178$.

4- Masci PG, Pussi A, Fontanive P, et al. Double chambered left ventricle in an asymptomatic adult patient. Eur Heart J Cardiovasc Imaging 2012;13:E1-3.

5- Zhang X, Cao T, Yuan L. Double-chambered left ventricle in echocardiography. Echocardiography $2011 ; 10: \mathrm{E} 67-68$.

6- Mordi I, Carrick D, Tzemos N. Diagnosis of double-chambered left ventricle using advanced cardiovascular imaging. Echocardiograpy. 2013;30:E206-208.

7- Sharma S, Dinwoodey D, Chaudhry G, et al. Congenital double-chambered left ventricle presenting as monomorphic ventricular tachycardia. CASE $2019 ; 3$ :51-55. 
8- Awad S, Patel A, Polimenakos A, et al. Left ventricular accessory chamber: a case report and review of the literature. Pediatr Cardiol 2009;30:1022-1025.

9- Ohlow MA, Von Korn H, Lauer B. Characteristics and outcome of congenital left ventricular aneurysm and diverticulum: Analysis of 809 cases published since 1816. Int J Cardiol $2015 ; 185: 34-45$.

\section{Legends}

Figures:

Figure 1 : Apical 4-chamber view at 24 weeks of pregnancy showing the accessory chamber (AC) and the main chamber $(\mathrm{MC})$ of the left ventricle separated by abnormal muscle bundle. LA: left atria, RA: right atria; RV: right ventricle.

Figure 2: Apical view showing that the main chamber (MC) of the left ventricle was in connection with mitral and aortic valves. AC: Accessory chamber; Ao: ascending aorta; LA: left atria; RV: right ventricle.

Figure 3: M-mode echocardiogram demonstrating that the accessory chamber (AC) of the left ventricle contracted synchronously with the main chamber (MC) and the right ventricle (RV).

Figure 4: M-mode color doppler showing bidirectional flow across the communication between accessory chamber (AC) and main chamber (MC) of the left ventricle. During diastole (D) red color indicates filling of the AC. During systole (S), blue color indicates draining of the AC towards the MC.

Figure 5: Postnatal subcostal view at 4 days of life confirming prenatal finding. AC: accessory chamber, LA: left atria; MC: main chamber; RA: right atria.

Figure 6 : Postnatal long axis parasternal view (A) at 4 days of life and (B) at 8 weeks. At 8 weeks, the left ventricle $(\mathrm{LV})$ is dilated with interventricular septum bowing to the right. Ao: ascending aorta; LA: left atria.

Videos:

Video 1: Apical view at 24 weeks showing the accessory chamber (AC) and main chamber (MC) of the left ventricle that lie side by side. AC has a normal contractility. LA: left atria; RA: right atria; RV: right ventricle.

Video 2: Apical view showing that the main chamber (MC) of the left ventricle is in connection with mitral valve and aortic valve. AC: accessory chamber; AO: ascending aorta; LA: left atria; RV: right ventricle.

Video 3: Postnatal echocardiogram apical view at 4 days of life demonstrating the antenatal findings. AC: accessory chamber; Ao: ascending aorta; LA: left atria; MC: main chamber; RV: right ventricle.

Video 4: Postnatal echocardiogram parasternal view at 8 weeks of life showing a dilated and hypokinetic left ventricle. 

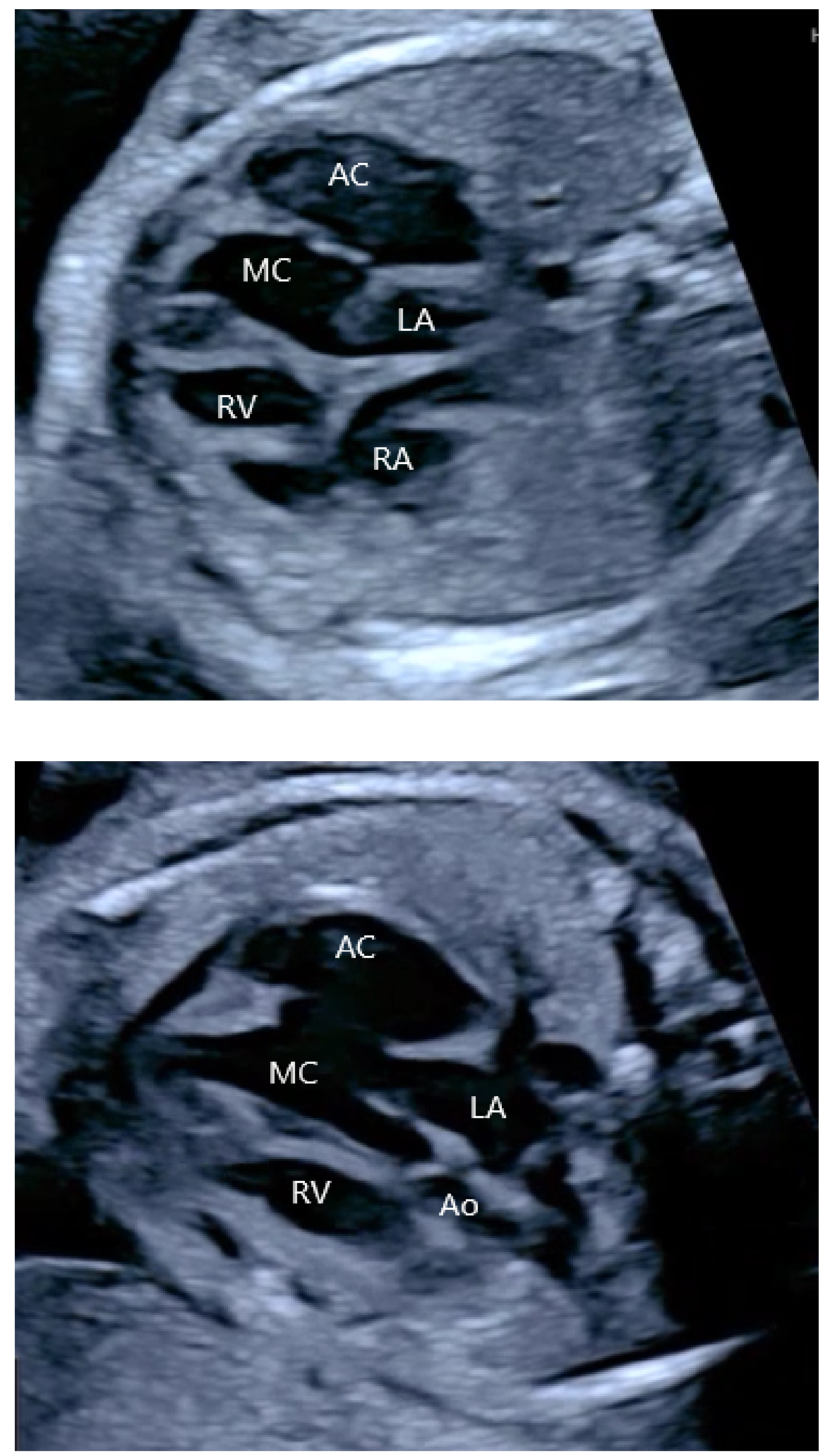

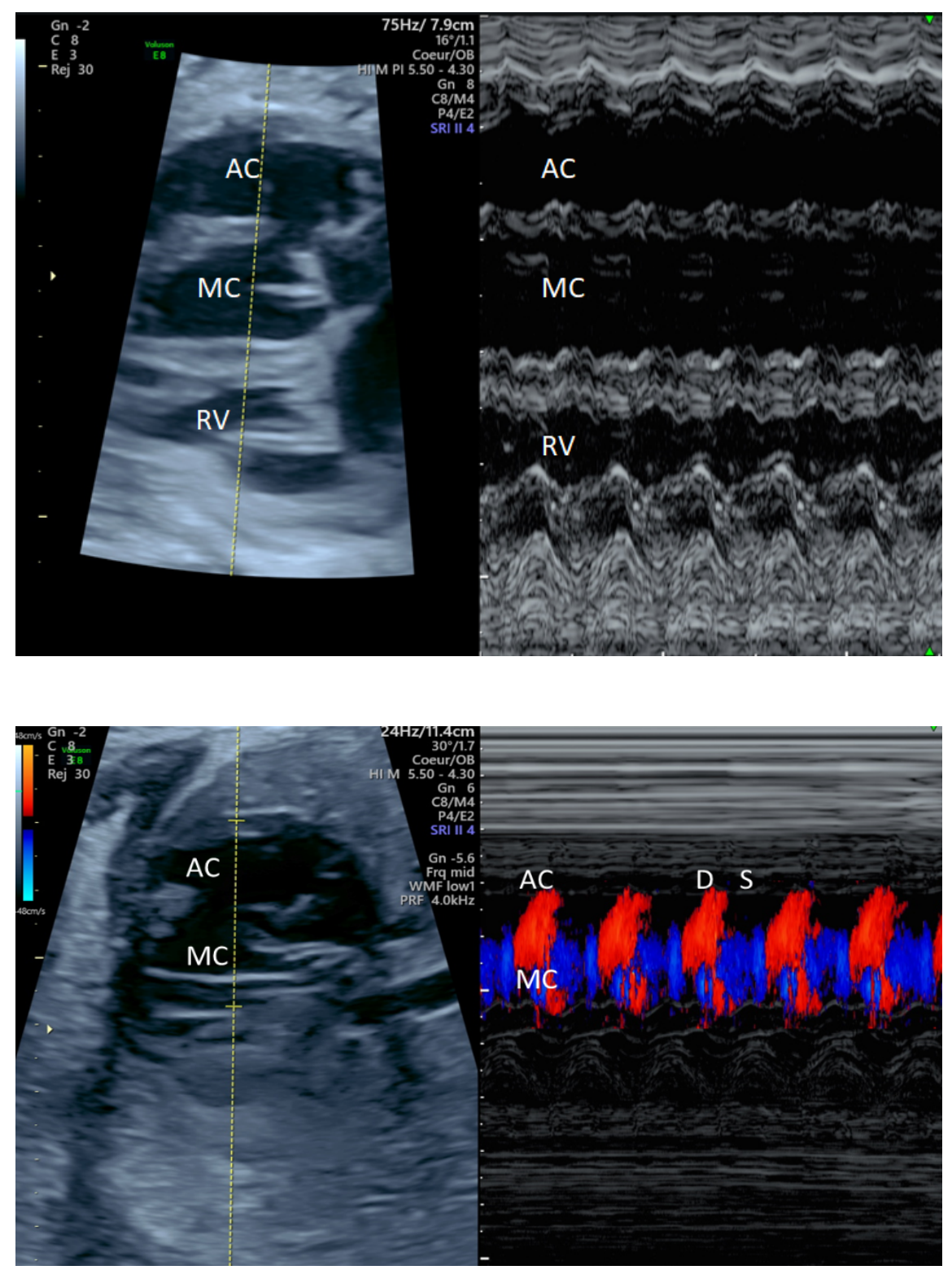

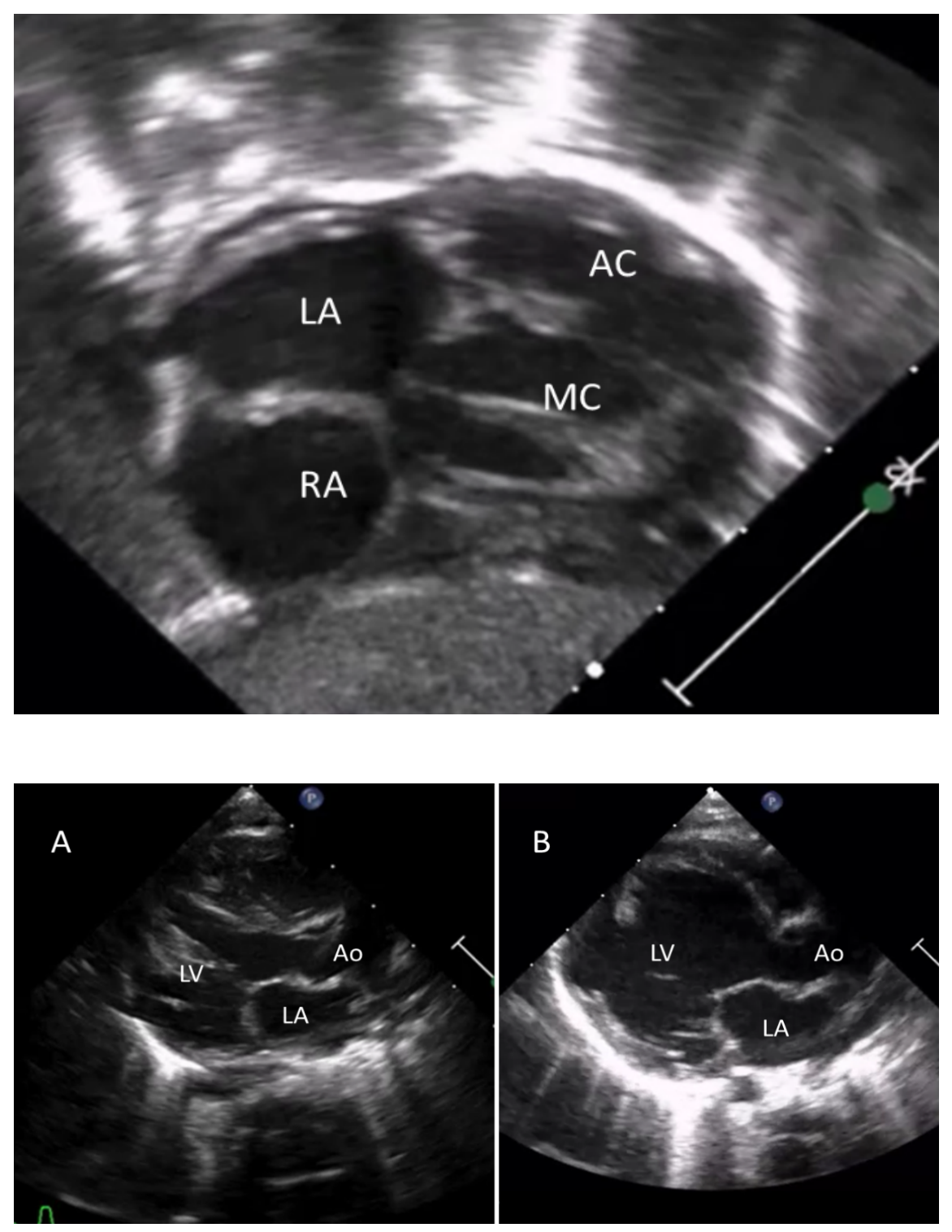